\title{
Feasibility and reproducibility of a cardiovascular magnetic resonance free-breathing, multi-shot, navigated image acquisition technique for ventricular volume quantification during continuous exercise
}

\author{
Pei G. Chew ${ }^{1}$, Peter P. Swoboda ${ }^{1}$, Carrie Ferguson ${ }^{2}$, Pankaj Garg ${ }^{1}$, Abigail L. Cook ${ }^{2}$, Said Ibeggazene ${ }^{2}$, \\ Louise A. E. Brown ${ }^{1}$, Thomas P. Craven ${ }^{1}$, James R. Foley ${ }^{1}$, Graham J. Fent ${ }^{1}$, Christopher E. Saunderson ${ }^{1}$, \\ David M. Higgins ${ }^{3}$, Sven Plein ${ }^{1}$, Karen M. Birch ${ }^{2}$, John P. Greenwood ${ }^{1}$ \\ ${ }^{1}$ Multidisciplinary Cardiovascular Research Centre \& Leeds Institute of Cardiovascular and Metabolic Medicine, University of Leeds, Leeds, UK; \\ ${ }^{2}$ School of Biomedical Sciences, University of Leeds, Leeds, UK; ${ }^{3}$ Philips, Guildford, UK
}

Correspondence to: Prof. John P. Greenwood, PhD. Multidisciplinary Cardiovascular Research Centre, Leeds Institute for Cardiovascular and Metabolic Medicine, University of Leeds, LS2 9JT, UK. Email: j.greenwood@leeds.ac.uk.

Background: Cardiovascular magnetic resonance (CMR) image acquisition techniques during exercise typically requires either transient cessation of exercise or complex post-processing, potentially compromising clinical utility. We evaluated the feasibility and reproducibility of a navigated image acquisition method for ventricular volumes assessment during continuous physical exercise.

Methods: Ten healthy volunteers underwent supine cycle ergometer (Lode) exercise CMR on two separate occasions using a free-breathing, multi-shot, navigated, balanced steady-state free precession cine pulse sequence. Images were acquired at 3-stages, baseline and during steady-state exercise at $55 \%$ and $75 \%$ maximal heart rate $\left(\mathrm{HR}_{\max }\right)$, based on a prior supine cardiopulmonary exercise test. Intra-and inter-observer variability and inter-scan reproducibility were derived. Clinical feasibility was tested in a separate cohort of patients with severe mitral regurgitation $(n=6)$.

Results: End-diastolic volume (EDV) of both LV and RV decreased during exercise at 55\% and $75 \%$ $\mathrm{HR}_{\max }$, although a reduction in RVEDV index was only observed at $75 \% \mathrm{HR}_{\max }$. Ejection fractions (EF) for both ventricles were significantly higher at $75 \% \mathrm{HR}_{\max }$ compared to their respective baselines (LVEF $68 \% \pm 3 \%$ vs. $58 \% \pm 5 \%, \mathrm{P}=0.001$; RVEF $66 \% \pm 4 \%$ vs. $58 \% \pm 7 \%, \mathrm{P}=0.02)$. Intra-observer and inter-observer reproducibility of $\mathrm{LV}$ parameters was excellent at all 3-stages. Although measurements of RVESV were more variable during exercise, the reproducibility of both RVEF and RV cardiac index was excellent (CV $<10 \%)$. Inter-scan LV and RV ejection fraction were highly reproducible at all 3 stages, although inter-scan reproducibility of indexed RVESV was only moderate. The protocol was well tolerated by all patients.

Conclusions: Exercise CMR using a free-breathing, multi-shot, navigated cine imaging method allows simultaneous assessment of left and right ventricular volumes during continuous exercise. Intra- and inter-observer reproducibility were excellent. Inter-scan LV and RV ejection fraction were also highly reproducible.

Keywords: Cardiovascular magnetic resonance (CMR); exercise; magnetic resonance imaging ergometer (MRI ergometer); free-breathing; feasibility; respiratory-navigation

Submitted Jan 15, 2020. Accepted for publication Jun 15, 2020.

doi: 10.21037/qims-20-117

View this article at: http://dx.doi.org/10.21037/qims-20-117 


\section{Introduction}

Exercise testing can be used to detect underlying cardiovascular abnormalities which are not apparent at rest. Whilst exercise-stress echocardiography and nuclear scintigraphy are widely available, their limitations include poor acoustic windows (1), motion artefacts (2) and radiation exposure (3). Cardiovascular magnetic resonance (CMR) imaging at rest is highly accurate and reproducible $(4,5)$, however exercise stress testing with CMR presents significant challenges. The early evolution of exercise CMR (exCMR) focused on improving the MRI-compatibility of exercise treadmill equipment from being placed external to the MRI room (6), to being in close proximity to the MRI scanner (7-9), to a fully MRI compatible treadmill placed adjacent to the MRI system $(8,10-12)$. These protocols are however limited by the time delay needed to transfer the patient from the treadmill onto the scanner. Any time delay between the cessation of exercise and MRI image acquisition is critical, since exercise-induced functional abnormalities may begin to disappear almost immediately after exercise cessation (13-15).

The development of a MRI-compatible cycle ergometer allows patients to exercise in the supine position whilst inside the bore of the magnet (16). Imaging during continuous exercise eliminates the time lapse between exercise and imaging and may allow a more accurate assessment of changes in cardiac physiology during exertion. Excessive motion during exercise however poses a challenge in image acquisition. As a result, investigators have resorted to acquire images following transient cessation of exercise $(17)$, during breath-holds $(6,17,18)$ or using ungated real-time cine imaging (19). Reconstruction of a short axis stack for volumetric analysis from ungated realtime imaging, however, involves complex post-processing analysis in addition to a requirement for bespoke in-house software (19).

The objectives of this study were: (I) to assess the feasibility and reproducibility of a navigated cine image acquisition method for the assessment of the ventricular volumes during continuous exercise; (II) to examine its clinical feasibility in patients with significant valvular heart disease.

\section{Methods}

\section{Study design and population}

This study was performed in 3 stages: (I) a pilot phase in which the feasibility of a navigated image acquisition 50 sequence was tested in healthy volunteers; (II) an assessment 51 of inter-scan reproducibility in which each healthy volunteer 52 underwent a repeat exCMR after a median of 16 weeks; (III) clinical application of this technique in patients with severe mitral regurgitation (MRegur). The study was approved by a local ethics committee (Yorkshire \& The Humber-Leeds West 12/YH/0551) and complied with the Declaration of Helsinki. All participants provided written informed consent.

\section{Pilot phase \& reproducibility}

Ten healthy volunteers with no history or symptoms of cardiovascular disease and no contraindications to CMR were recruited. Absolute and relative contraindications to exercise testing were adhered to according to American Heart Association (AHA) guidelines (20). All participants had a height of $<190 \mathrm{~cm}$. All healthy volunteers underwent a supine cardiopulmonary exercise test (CPET) prior to undertaking exCMR on a supine cycle ergometer. CMR was performed on a 1.5 Tesla MRI system with $70 \mathrm{~cm}$ bore (Ingenia, Philips Healthcare, Best, Netherlands) equipped with a 28-channel coil and free-breathing images were acquired during continuous exercise. Exercise intensity was individualized to the heart rate (HR) corresponding to $55 \%$ and $75 \%$ of the maximal $\mathrm{HR}\left(\mathrm{HR}_{\max }\right)$ attained on their pre-CMR supine CPET. After a median time of 16 weeks, exCMR was repeated using an identical scanner and protocol.

\section{Clinical feasibility}

The potential for translation of this technique into clinical practice was examined in a separate cohort of 6 patients with significant MRegur, all prospectively recruited from the valvular heart disease clinic at Leeds Teaching Hospitals NHS Trust. Inclusion criteria included: moderate-severe or severe MRegur on echocardiography, and New York Heart Association functional Class I. Exclusion criteria included: contraindications to exercise stress testing according to AHA guidelines (20), presence of atrial fibrillation, height $>190 \mathrm{~cm}$, inability to exercise and contraindications to CMR. In our institution treadmill CPET is used clinically in patients with significant MRegur and we utilized these data to prescribe the individualized HR during exCMR. To allow for the lower HR response in supine cycling compared to upright treadmill exercise and the reduced 
exercise tolerance seen in patients with severe MRegur, the prescribed HR had to be altered from healthy volunteers. Patients were thus exercised to $30-39 \%$ and $40-59 \%$ of their heart rate reserve (HRR), corresponding to 'light' and 'moderate'-intensity exercise according to the American College of Sports Medicine guidelines (21). HRR was calculated based on this formula: resting HR on CPET + [ $x \%$ of (max HR achieved on treadmill CPET - resting HR)]; where $x$ is the target $\%$ of HRR.

\section{Cardiopulmonary exercise testing}

All healthy volunteers underwent CPET on a supine cycle ergometer (Lode BV, Groningen, The Netherlands). The crank length on the pre-CMR cycle ergometer was adjusted to replicate the setup of the in-scanner MRI ergometer. CPET was conducted as a ramp incremental test $(15 \mathrm{~W} / \mathrm{min})$ to volitional intolerance. Breath-by-breath analysis of the volume and concentration of expired gases was achieved using an automated system (Medgraphics Ultima, Minnesota, USA). HR was continuously monitored via an attached 12-lead electrocardiogram (ECG). The main outcome measures were maximal HR and maximal power output in Watts. ExCMR was performed after a median of 8 days [interquartile range (IQR) 2-13].

\section{Exercise CMR protocol and image acquisition}

Exercise whilst in the bore of the magnet was conducted on a supine MRI-compatible cycle ergometer (Lode BV, Groningen, The Netherlands). Optimal participant preparation included instructions on consistent thoracic breathing, use of handrail to ensure trunk stability, skin preparation to maximize interface between electrode and skin, and securing vector ECG connections onto anterior chest wall with tape to ensure quality recording of ECG. A blood pressure (BP) cuff was placed on the left arm. Both the surface coil and torso pad were then firmly secured onto the participants with elastic Velcro ${ }^{\circledR}$ straps. The MRI table was advanced whilst participants performed a short bout of unloaded exercise to ensure that their knees did not contact the scanner casing during pedalling.

Free-breathing images were acquired at 3 -stages, at rest and then during steady-state exercise at $55 \% \mathrm{HR}_{\max }$ and $75 \% \mathrm{HR}_{\max }$. Exercise began with a 2 min warmup at a power output of $0 \mathrm{~W}$ (unloaded). Work rate was incrementally increased by $10-20 \mathrm{~W}$ until the target $55 \%$ $\mathrm{HR}_{\max }$ was achieved, and then adjusted to maintain the
$\mathrm{HR}$ at the required target throughout the exercise. Verbal 146 feedback was constantly given to participants and cycling 147 cadence was maintained between 60-70 rpm. Following 148 a rest period of 2 minutes, a second bout of exercise was 149 undertaken until the target $75 \% \mathrm{HR}_{\max }$ was achieved. 150 Heart rate and rhythm were continuously monitored, and 151 $\mathrm{BP}$ was recorded at each stage. Each stage of exercise was 152 maintained for 5-7 minutes ( 2 minutes to achieve steady- 153 state in HR and approximately 3-5 minutes of image 154 acquisition). Imaging was only performed during steady- 155 state conditions, when HR was maintained at near constant 156 levels. Criteria for termination prior to achieving target $\quad 157$ HR included participant's request and a drop in systolic BP $>10 \mathrm{mmHg}$.

The scan protocol included standard long axis views (vertical, horizontal long axis) and a short axis ventricular volume stack. Cine imaging was performed using a freebreathing, multi-shot, respiratory-navigated, balanced steady-state free precession pulse sequence. A respiratory echo-based navigator was placed on the right hemidiaphragm with a $5 \mathrm{~mm}$ gating window and continuous gating level drift activated. A cylindrical MR radiofrequency excitation pulse from which a 1-dimensional projection of the lung-liver interface was generated and was used to infer the breathing phase. The navigator was played at the start of the R-R interval, at end-diastole of the cardiac cycle. The steady-state of ongoing balanced steady-state free precession (bSSFP) readout was stopped in the standard controlled manner by using half-alpha radiofrequency pulses to temporarily store the steady state magnetization in the z-direction. This allowed the respiratory navigator to last for a total duration of $24 \mathrm{~ms}$ (played out for $17 \mathrm{~ms}$ before resuming readout after $7 \mathrm{~ms}$ ), equivalent to approximately 9 repetition time (TR). Retrospective cardiac triggering was used in this study (continuous data sampling). The bSSFP readout was continuous, wherein data from the ECG and k-space profile acquisition timings were matched to produce images for all cardiac phases. Cartesian sampling was used, and the acquired $\mathrm{k}$-space lines were only accepted for image reconstruction if the right hemi-diaphragm position was within the gating window during end-expiratory phase. K-space profiles which were rejected outside of the respiratory navigator gate were reacquired. Other scan parameters were as follows: typical field of view (FOV) $320 \mathrm{~mm} \times 320 \mathrm{~mm}$, repetition time (TR) $2.8 \mathrm{~ms}$, echo time (TE) $1.4 \mathrm{msec}$, flip angle $60^{\circ}$, temporal resolution $33 \mathrm{~ms}$, SENSE factor 2, multi-shot turbo field echo (TFE) factor 11, TFE acquisition duration $30.4 \mathrm{ms,}$ 156 47 . 1

\section{3}

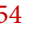
is 

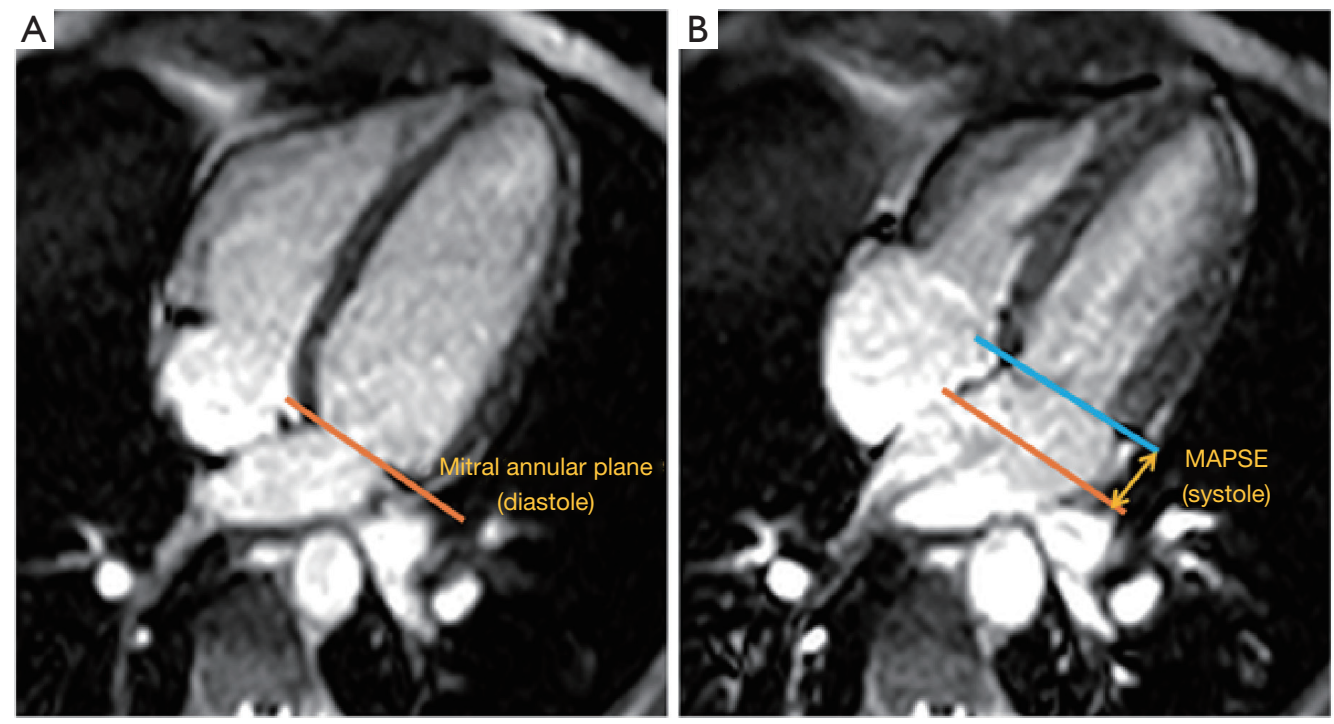

Figure 1 Assessment of longitudinal function. (A) End-diastole phase was identified and a refence line (orange) was drawn across the atrioventricular valve plane and forwarded across all phases of the cine image. (B) A further line (blue) is drawn in end-systole. The distance between the two points at the lateral mitral valve annulus (MAPSE) was measured and expressed in mm. MAPSE, mitral annular plane systolic excursion.

phase percentage $50 \%$, slice thickness $10 \mathrm{~mm}, 0 \mathrm{~mm}$ gap, in-plane spatial resolution $2.4 \mathrm{~mm} \times 2.4 \mathrm{~mm}$ and matrix $132 \times 106$. A total of 16 cardiac phases were acquired and this was reconstructed to 30 cardiac phases.

\section{CMR analysis}

CMR analysis was performed by two independent operators (PC, LB; both observers with 3 years CMR experience) using commercially available computer software $\left(\mathrm{cmr}^{42}\right.$, Circle Cardiovascular Imaging Inc, Calgary, Alberta, Canada). Left and right ventricular volumes, and ejection fraction (EF) were calculated in the conventional method, by manually tracing endocardial contours in end-diastole and end-systole on the short axis stack (Figure S1). Biventricular end-diastolic and end-systolic volumes were calculated using a summation of discs technique (22). Stroke volume (SV) was measured as the difference between enddiastolic and end-systolic volume, whereas cardiac output was calculated as: SV $\times$ HR. All measured volumes and cardiac output parameters were indexed to body surface area (Mostellar formula). Longitudinal LV function in the form of mitral annular plane systolic excursion (MAPSE) was assessed by using mitral annular excursion. In the 4-chamber cine image, atrioventricular motion was measured at the lateral junction points between the left atrium and ventricle at end diastole and end systole. The perpendicular distance between these two points was measured. Figure 1 outlines the methodology used to assess LV longitudinal contraction.

\section{Statistical analysis}

All statistical analysis was performed using the SPSS V.21.0 (IBM Corp., New York, USA). All continuous data were tested for normality using the Shapiro-Wilk test; variables are expressed as mean $\pm \mathrm{SD}$ or median (IQR) in cases of skewed distributions. Categorical variables are expressed as frequencies and percentages. Repeated measures analysis of variance (ANOVA) with Bonferroni post-test analysis was used to compare data between rest and different stages of exercise. Intra- and inter-observer reproducibility was assessed by the coefficient of variation $(\mathrm{CV})$ test, the standard deviation of differences between observations divided by the mean. $\mathrm{P}<0.05$ was considered statistically significant.

\section{Results}

Healthy volunteers and baseline CMR data

All 10 healthy volunteers [ 7 men, age $25 \pm 2$ years, body 
Table 1 Volumetric data at baseline, and during exercise at $55 \%$ and $75 \% \mathrm{HR}_{\max }$ in healthy volunteers

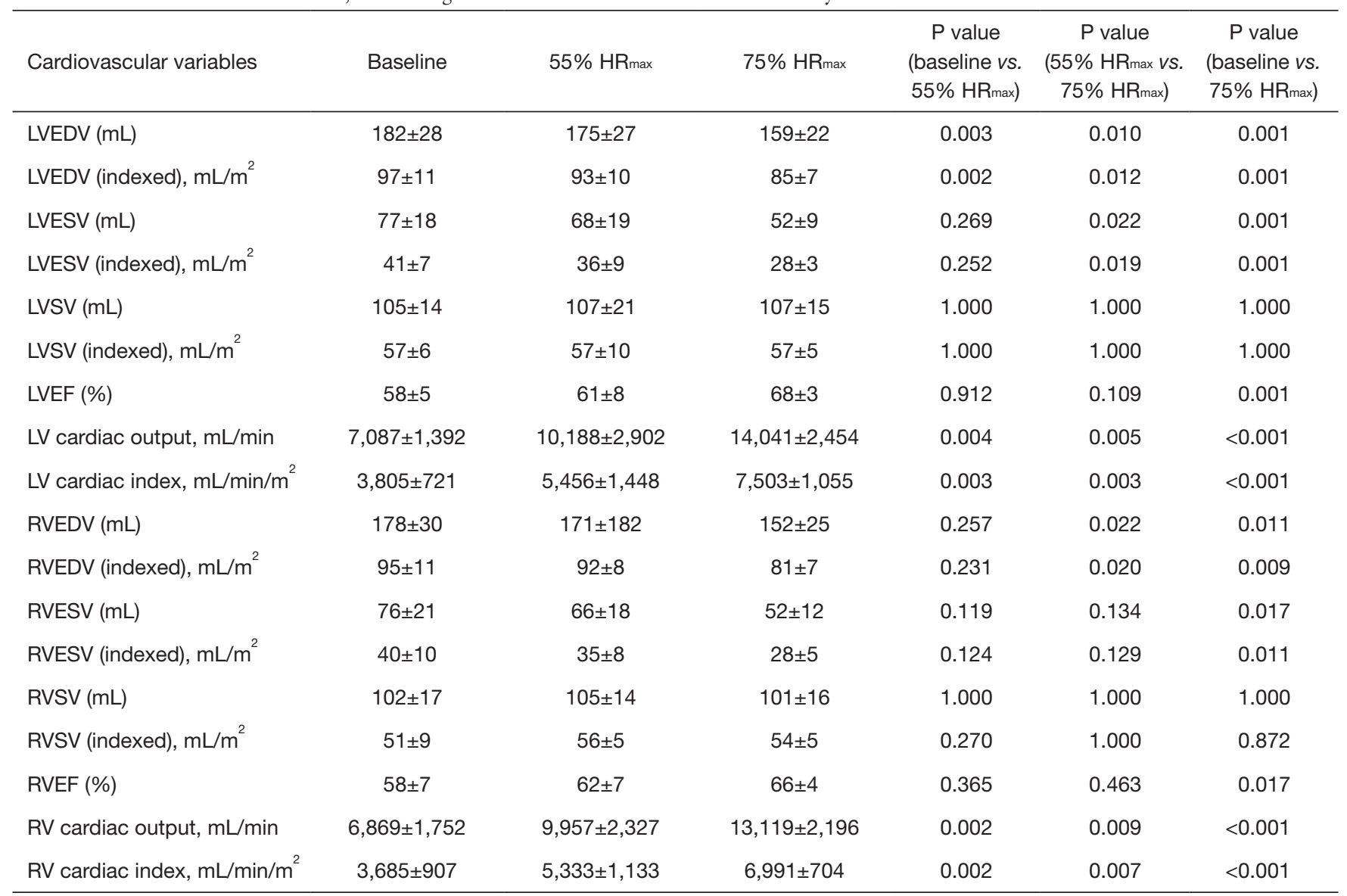

Data as mean \pm SD. LVEDV, left ventricular end-diastolic volume; LVESV, left ventricular end-systolic volume; LVSV, left ventricular stroke volume; LVEF, left ventricular ejection fraction; LV, left ventricle; RVEDV, right ventricular end-diastolic volume; RVESV, right ventricular endsystolic volume; RVSV, right ventricular stroke volume; RVEF, right ventricular ejection fraction; RV, right ventricle.

mass index (BMI) $\left.23.1 \pm 2.2 \mathrm{~kg} / \mathrm{m}^{2}\right]$ completed the full study protocol. HR increased during exercise $(68 \pm 12$ vs. $94 \pm 13$ vs. $131 \pm 11 \mathrm{bpm}$, baseline vs. $55 \% \mathrm{HR}_{\max }$ vs. $75 \% \mathrm{HR}_{\max }$; all $\mathrm{P}<0.001)$. Systolic BP was significantly higher during exercise at $75 \% \mathrm{HR}_{\max }$ than at baseline $(130 \pm 12$ vs. $120 \pm$ $10 \mathrm{mmHg} ; \mathrm{P}=0.03)$, whilst diastolic $\mathrm{BP}$ remained unchanged (70 14 vs. $70 \pm 8 \mathrm{mmHg} ; \mathrm{P}=1.00)$. Mean supine work rate for exercise at $55 \% \mathrm{HR}_{\max }$ and $75 \% \mathrm{HR}_{\max }$ was $25 \pm 19 \mathrm{~W}$ and $87 \pm 23 \mathrm{~W}$, respectively. CMR data for all subjects are shown in Table 1. Figure 2 demonstrated the exCMR images at baseline and during exercise.

\section{Left ventricular $(L V)$ and right ventricular $(R V)$ parameters during exercise}

The changes in ventricular volumes during exercise are plotted in Figure 3. End-diastolic volume (EDV) of the LV 261 decreased significantly during exercise at $55 \%$ and $75 \% \quad 262$ $\mathrm{HR}_{\max }$. In contrast, RVEDV remained unchanged from 263 baseline at $55 \% \mathrm{HR}_{\max }$ and significantly decreased at $75 \% 264$ $\mathrm{HR}_{\max }(\mathrm{P}=0.02) . \mathrm{LV}$ end-systolic volume (LVESV) decreased 265 when exercised from $55 \% \mathrm{HR}_{\max }$ to $75 \% \mathrm{HR}_{\max }(\mathrm{P}=0.02) . \quad 266$ During exercise at $55 \% \mathrm{HR}_{\max }$, LVESV was however 267 not significantly different from baseline. RV end-systolic 268 volume (RVESV) significantly decreased during exercise at 269 $75 \% \mathrm{HR}_{\max }$ compared to baseline. Both LV and RV stroke 270 volumes remained unchanged. Ejection fractions (EF) for 271 both ventricles were significantly higher during exercise 272 at $75 \% \mathrm{HR}_{\max }$ when compared to their respective baseline 273 values (LVEF $68 \% \pm 3 \%$ vs. $58 \% \pm 5 \% ; \mathrm{P}=0.001$ and $\mathrm{RVEF} 274$ $66 \% \pm 4 \%$ vs. $58 \% \pm 7 \% ; \mathrm{P}=0.02)$. During exercise, $\mathrm{LV}$ and 275 RV cardiac indexes also increased significantly (Figure 4). $\quad 276$ 

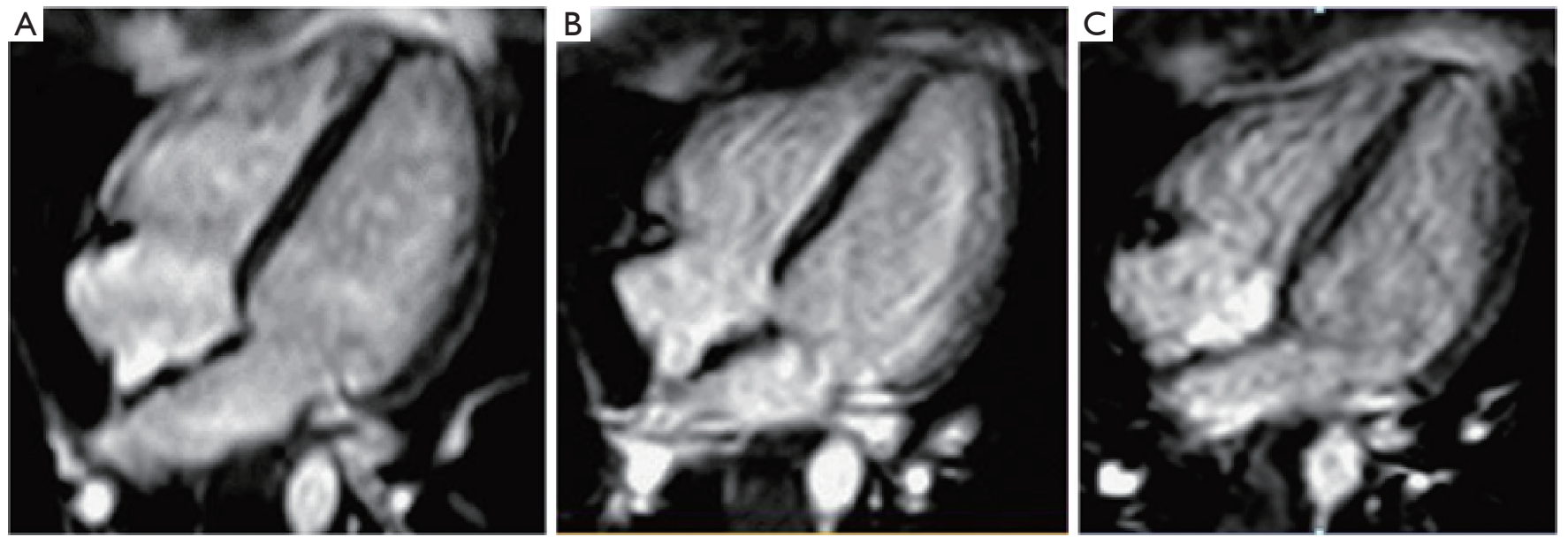

Figure 2 exCMR images at baseline (A), and during exercise at $55 \% \mathrm{HR}_{\max }(\mathrm{B})$ and $75 \% \mathrm{HR}_{\max }(\mathrm{C}) . \mathrm{HR}_{\max }$ maximal heart rate.
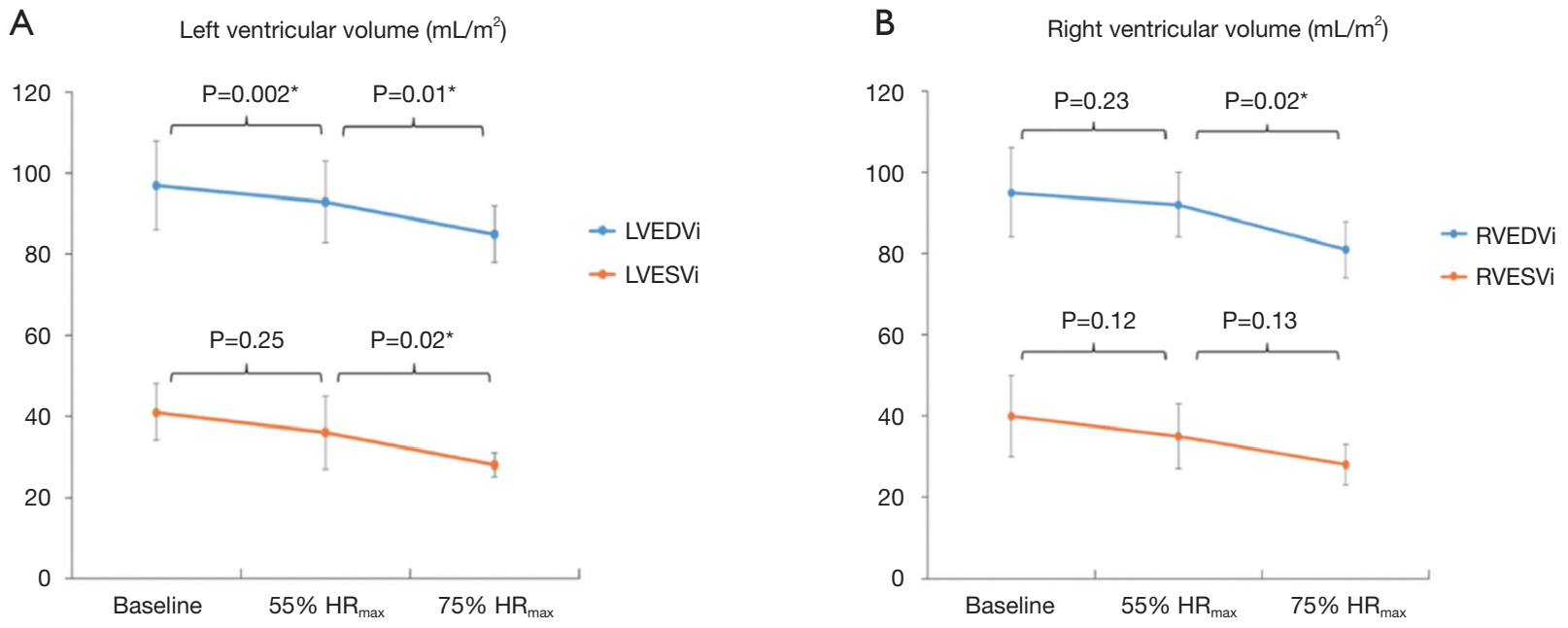

Figure 3 Ventricular volumes during exercise in healthy volunteers. LV (A) and RV (B) end-diastolic and end-systolic volumes during exercise in healthy volunteers. LVEDVi, indexed LV end-diastolic volume; LVESVi, indexed LV end-systolic volume; RVEDVi, indexed right ventricular end-diastolic volume; RVESVi, indexed right ventricular end-systolic volume; $\mathrm{HR}_{\max }$, maximal heart rate.

\section{LV longitudinal contraction}

Baseline MAPSE appears to be higher in healthy volunteers when compared to patients with severe mitral regurgitation $(14 \pm 4$ vs. $12 \pm 3 \mathrm{~mm}$ ) (Figure 5). In the healthy volunteers, MAPSE increased from $14 \pm 4$ to $19 \pm 5 \mathrm{~mm}(\mathrm{P}=0.05)$ during exercise at $55 \% \mathrm{HR}_{\max }$. At $75 \% \mathrm{HR}_{\max }$, MAPSE appears to decrease to $17 \pm 4 \mathrm{~mm}$ although this change was not statistically significant $(\mathrm{P}=1.00)$. In patients with severe mitral regurgitation, there was no significant change of MAPSE between baseline and both stages of exercise.
There is a trend however, indicating that MAPSE increased with exercise and appear to decline slightly when higher intensity exercise was achieved.

\section{Intra- and inter-observer reproducibility}

Intra-observer reproducibility of $\mathrm{LV}$ volumes, $\mathrm{LV}$ ejection fraction and LV cardiac index was excellent at all three stages, evidenced by $\mathrm{CV} \leq 10 \%$ (Table 2). During exercise, the measurements of RVESV were more variable $(\mathrm{CV}$ 11-20\%). The reproducibility of RV EDV, RV ejection 
A

LV ejection fraction (\%)

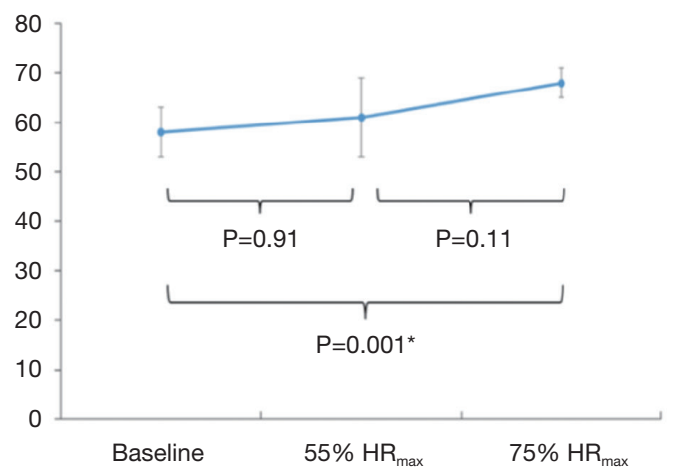

C

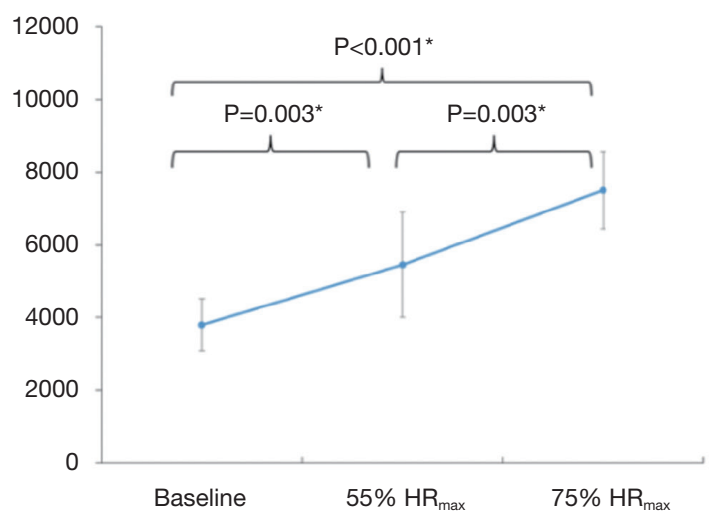

B

RV ejection fraction (\%)

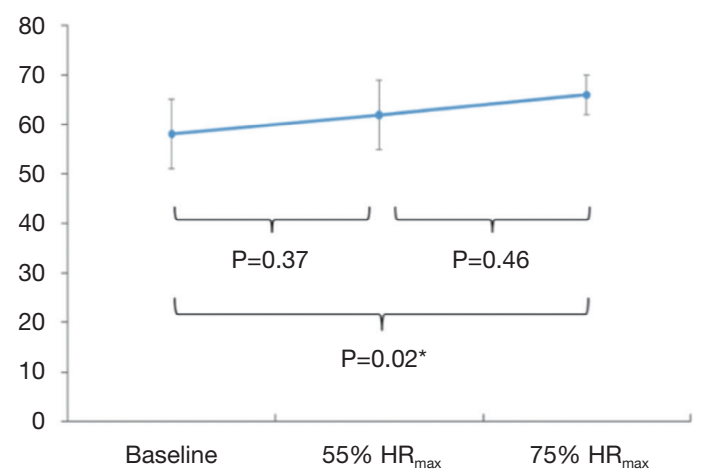

D RV cardiac index $\left(\mathrm{mL} / \mathrm{min} / \mathrm{m}^{2}\right)$

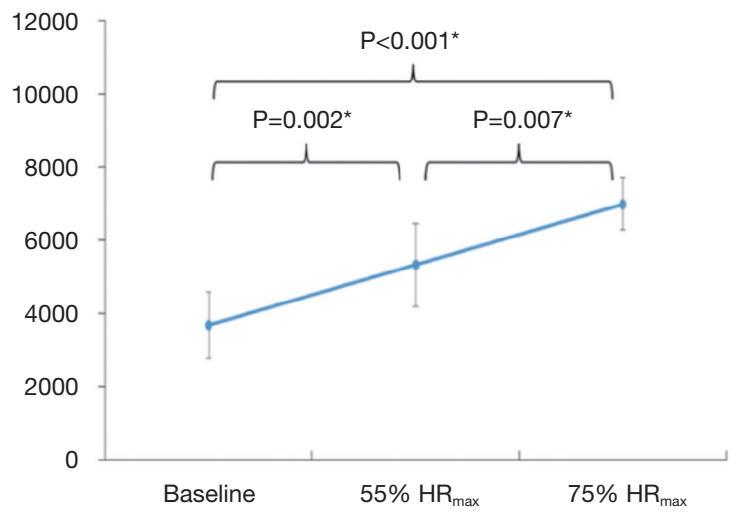

Figure 4 Exercise cardiac reserve in healthy volunteers. (A,B) LV and RV ejection fraction; (C,D) LV and RV cardiac indexes during exercise in healthy volunteers. Data presented in mean (dots) and standard deviation (bars). Asterisks denote statistically significant differences $(\mathrm{P}<0.05) . \mathrm{HR}_{\max }$, maximal heart rat.

\begin{tabular}{|c|c|c|c|c|c|c|c|}
\hline Group & $\begin{array}{l}\text { MAPSE } \\
\text { (baseline) }\end{array}$ & $\begin{array}{c}\text { MAPSE } \\
\left(55 \% \mathrm{HR}_{\max }\right)\end{array}$ & $\begin{array}{c}\text { MAPSE } \\
\left(75 \% \mathrm{HR}_{\max }\right)\end{array}$ & $\begin{array}{c}\text { P value (baseline } \\
\left.\text { vs. } 55 \% \mathrm{HR}_{\max }\right)\end{array}$ & $\begin{array}{c}\text { P value } \\
\text { (55\% HRmax vs. } \\
\left.75 \% \mathrm{HR}_{\max }\right)\end{array}$ & $\begin{array}{c}\text { P value } \\
\text { (baseline vs. } \\
75 \% \mathrm{HR}_{\max } \text { ) }\end{array}$ & $\begin{array}{l}\text { ANOVA with } \\
\text { Bonferroni } \\
\text { correction }\end{array}$ \\
\hline Group & $\begin{array}{l}\text { MAPSE } \\
\text { (baseline) }\end{array}$ & $\begin{array}{l}\text { MAPSE (light } \\
\text { intensity) }\end{array}$ & $\begin{array}{l}\text { MAPSE } \\
\text { (moderate } \\
\text { intensity) }\end{array}$ & $\begin{array}{c}\text { P value (baseline } \\
\text { vs. light) }\end{array}$ & $\begin{array}{l}\text { P value (light vs. } \\
\text { moderate) }\end{array}$ & $\begin{array}{c}\text { P value } \\
\text { (baseline vs. } \\
\text { moderate) }\end{array}$ & $\begin{array}{c}\text { ANOVA with } \\
\text { Bonferroni } \\
\text { correction }\end{array}$ \\
\hline
\end{tabular}

Figure 5 Longitudinal contraction in both healthy volunteers and patients with severe mitral regurgitation. Data as mean \pm SD. MAPSE, mitral annular plane systolic excursion; MR, mitral regurgitation. 
fraction, and RV cardiac index was however excellent (CV $<10 \%)$.

Inter-observer reproducibility of $\mathrm{LV}$ volumes, $\mathrm{LV}$ ejection fraction and LV cardiac index was also excellent at all three stages (CV for LVEDV $\leq 5 \%$; LVESV $\leq 10 \%$; LVEF $<6 \%$; LV cardiac index $<8 \%)$. With incremental exercise, inter-observer reproducibility was better in the assessment of RVEDV (CV $<5 \%)$, when compared to RVESV measurements (CV 12-14\%). Although measurements of RVESV were more variable during exercise, the reproducibility of RV ejection fraction, RV stroke volume and RV cardiac index was however excellent. During exercise at $75 \% \mathrm{HR}_{\max }$, inter-observer LVESV was more reproducible than RVESV (CV 10\% vs. 14\%).

\section{Inter-scan reproducibility}

We observed good inter-scan reproducibility for LV enddiastolic and end-systolic volumes during exercise; although only modest reproducibility was seen in the readings of $\mathrm{LV}$ cardiac index (CV 10-16\%). The RVESV measurements were the least reproducible (CV 11-24\%). Inter-scan LV and $\mathrm{RV}$ ejection fraction were however highly reproducible $(\mathrm{CV}<10 \%)$ at all 3 stages.

\section{Ventricular volumes in clinical patients}

Of 6 patients with severe MRegur, 5 patients (60\% men, age $60 \pm 14$ years, BMI $24 \pm 2.2 \mathrm{~kg} / \mathrm{m}^{2}$ ) completed the full study protocol. exCMR had to be abandoned in 1 patient due to a significant hypotensive response. HR increased throughout exercise $(73 \pm 6$ vs. $111 \pm 11$ vs. $118 \pm 18 \mathrm{bpm}$, baseline vs. light vs. moderate; all $\mathrm{P}<0.01)$. Systolic $\mathrm{BP}$ was significantly higher during moderate intensity exercise than at baseline (114 \pm 6 vs. $148 \pm 15 \mathrm{mmHg} ; \mathrm{P}=0.02)$, whilst diastolic $\mathrm{BP}$ remained constant $(74 \pm 9$ vs. $80 \pm 8$ vs. $66 \pm 14 \mathrm{mmHg}$; $\mathrm{P}=1.00$ ). Mean supine work rate for light and moderateintensity exercise was $44 \pm 19$ and $53 \pm 32 \mathrm{~W}$, respectively. CMR data for all clinical patients are described in Table 3. There was no significant change in the LVEDV during exercise in this small patient sample, and despite a downward trend of LVESV, this was not significant. LVEF was significantly higher when moderate-intensity exercise was achieved. The augmentation of cardiac output and cardiac index was apparent with incremental exercise. When considering the RV parameters, there was no significant change in its EDV. During moderate-intensity exercise, RVESV was significantly smaller than at baseline.
Despite a numerical increase in RVEF with exercise, this 356 was not significant. RV cardiac output and RV cardiac index 357 were unchanged during light-intensity exercise but were 358 significantly increased during moderate-intensity exercise.

\section{Discussion}

This study demonstrated the (I) feasibility of the freebreathing, multi-shot, navigated image acquisition method in the serial assessment of ventricular volumes during continuous exercise; (II) excellent intra- and inter-observer reproducibility, in particular the LV indices; (III) clinical feasibility of this imaging method in a challenging group of patients with significant mitral regurgitation, the first exCMR study performed in this patient group.

Previously, image acquisition techniques using the MRI cycle ergometer have either involved a brief period of exercise cessation (17) or required a breath-hold protocol $(6,18)$ in order to reduce excessive motion artefacts and avoid poor ECG signal. Ungated real-time CMR imaging $(19,23,24)$ has been a method that enabled cine images to be acquired during continuous exercise. However, the postprocessing analysis of these images requires retrospective synchronization of ECG and respiratory movements, in addition to the need for non-commercially available inhouse software (19), therefore decreasing widespread attainability. The application of other image acquisition techniques such as motion correction $(25,26)$ can be challenging in this setting due to the large amount of through plane motion during exercise. Navigator-echobased gating techniques have been practical methods for effective reduction of respiration motion effects, and are well established for coronary MRI imaging $(27,28)$. Our feasibility study demonstrated that the application of respiratory-navigated technique in exCMR has the potential to overcome respiratory motion which can be quite significant during vigorous exercise. This technique was feasible in both healthy volunteers and clinical patients, and the images acquired were analyzable and reproducible. Moreover, this imaging technique allowed serial assessment of cardiac function at incremental exercise with a further advantage that image analysis can be performed on widely used, commercially available software. This protocol therefore has the potential to increase the utility of exCMR as a clinical assessment tool.

La Gerche et al. (19) compared real-time ungated with gated CMR techniques and demonstrated that despite its complex post-processing analysis, ventricular volumes 
Table 2 Coefficient of variation (CV) for the reproducibility of LV and RV cardiac indices

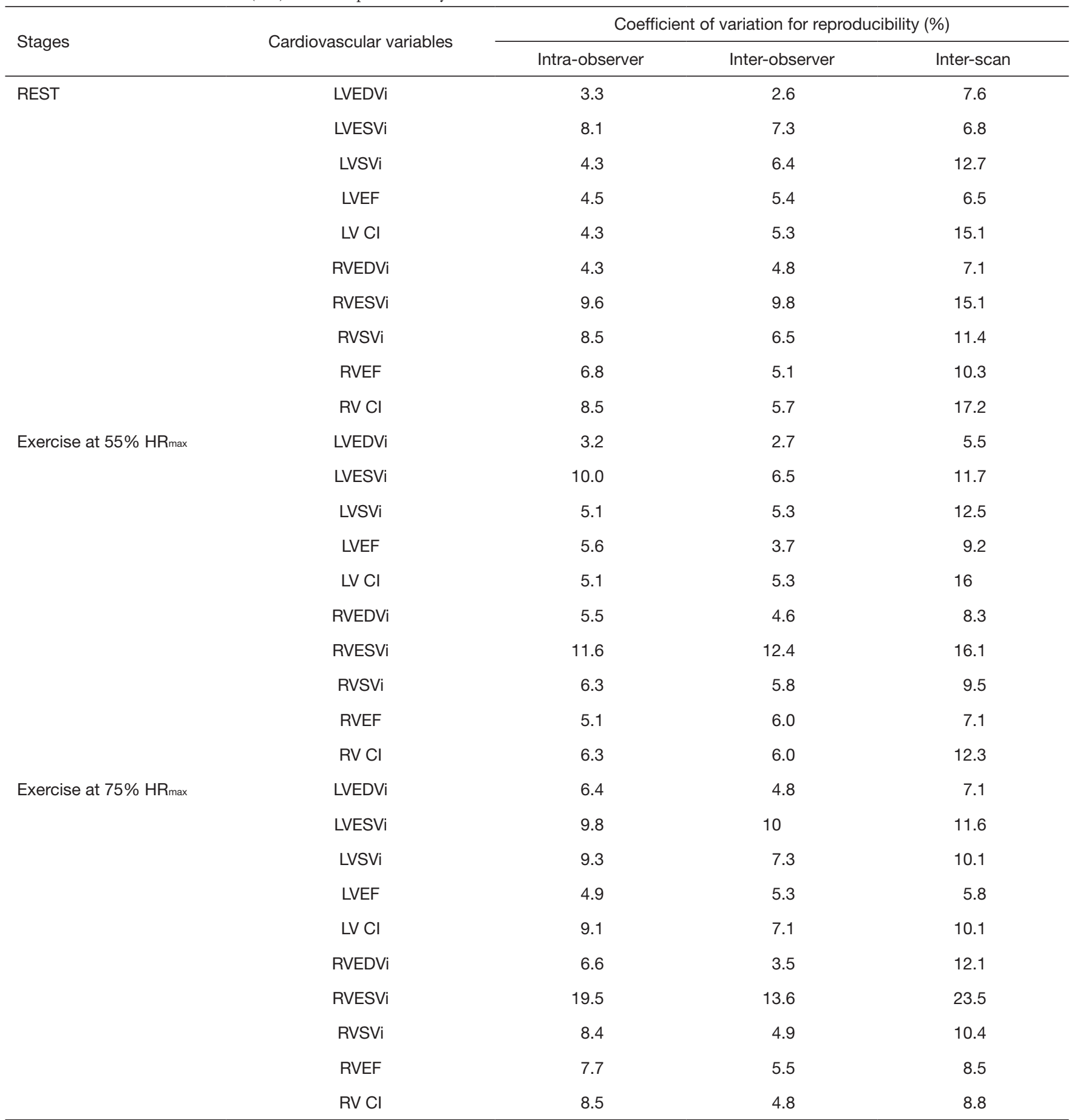

Data as \%. HRmax, maximal heart rate; LVEDVi, indexed left ventricular end-diastolic volume; LVESVi, indexed left ventricular end-systolic volume; LVSVi, indexed left ventricular stroke volume; LVEF, left ventricular ejection fraction; LV Cl, left ventricular cardiac index; RVEDVi, indexed right ventricular end-diastolic volume; RVESVi, indexed right ventricular end-systolic volume; RVSVi, indexed right ventricular stroke volume; RVEF, right ventricular ejection fraction; RV CI, right ventricular cardiac index; HR, heart rate. 
Table 3 Volumetric data at baseline, and during light and moderate-intensity exercise in clinical patients

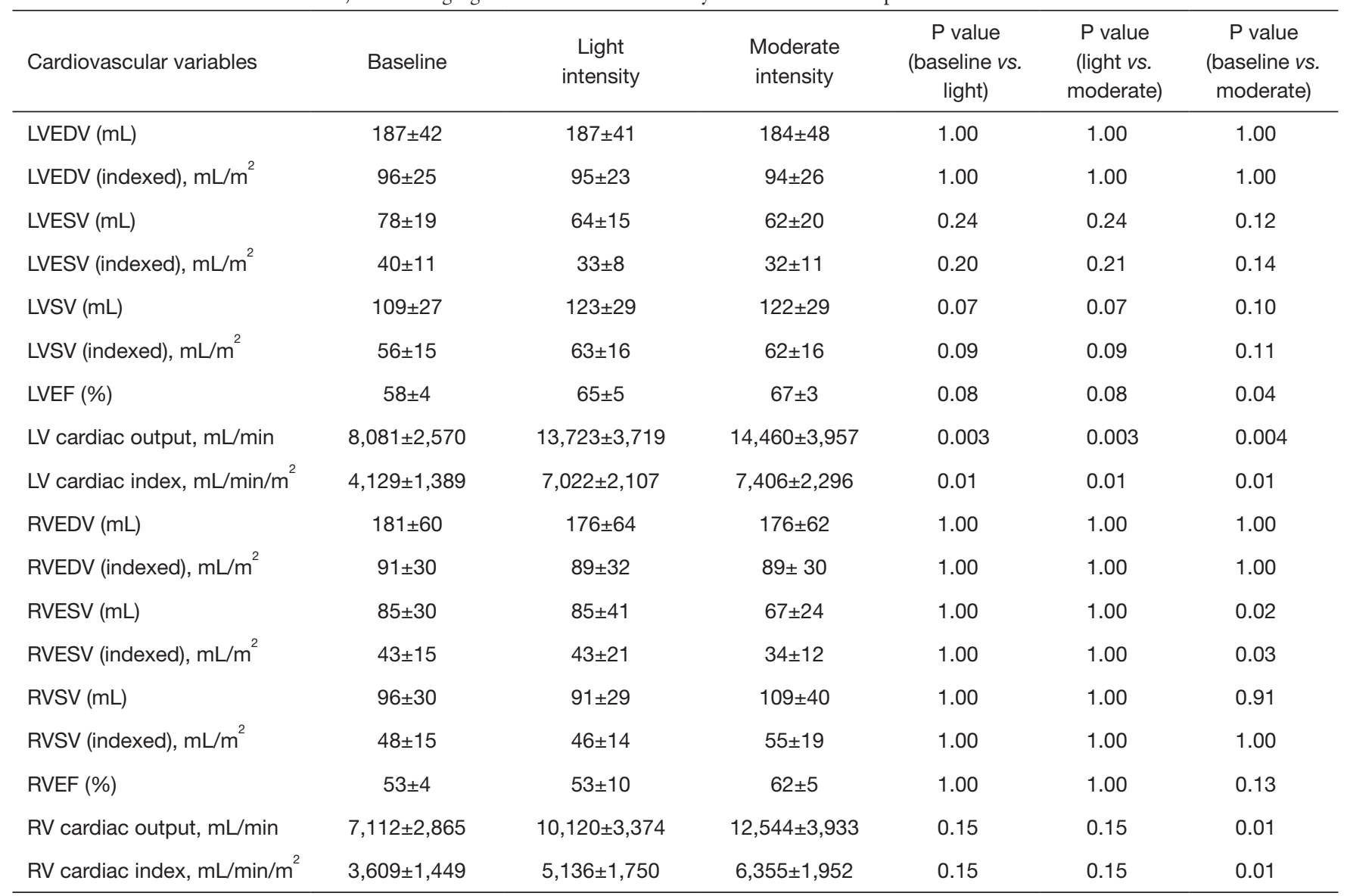

Data as mean \pm SD. LVEDV, left ventricular end-diastolic volume; LVESV, left ventricular end-systolic volume; LVSV, left ventricular stroke volume; LVEF, left ventricular ejection fraction; LV, left ventricle; RVEDV, right ventricular end-diastolic volume; RVESV, right ventricular endsystolic volume; RVSV, right ventricular stroke volume; RVEF, right ventricular ejection fraction; RV, right ventricle.

were analysable more frequently with real-time ungated compared with gated CMR (100\% vs. $47 \%$; $\mathrm{P}<0.001)$. In our gated CMR study, when combined with 'respiratorynavigation', sufficient image quality for analysis was achieved in $100 \%$ of the scans. La Gerche et al. also observed better interobserver variability for real-time ungated $(\mathrm{CV}=1.9 \%$ and $2.0 \%$ for $\mathrm{LV}$ and $\mathrm{RV}$ stroke volumes, respectively) than gated scans $(C V=15.2 \%$ and $13.6 \% ; \mathrm{P}<0.01)(19)$. Comparing their gated study to ours, the incorporation of 'respiratory-navigation' in our gated study improved the CV for left and RV stroke volumes (CV of 7.3\% and 4.9\%, during exercise at $75 \% \mathrm{HR}_{\max }$, respectively).

In 2017, Le et al. (24) combined real-time imaging with ECG-gated sequences to assess exercise cardiac volumetrics in healthy volunteers and athletes. Image acquisition, however, required suspension of exercise at the end of every stage for free-breathing imaging. The decline in 419 HR following cessation of exercise $(15,29)$ can potentially 420 impair diagnostic accuracy and clinical utility. In contrast, 421 our exCMR protocol permits imaging during continuous 422 exercise, eliminating the time lapse between exercise and 423 imaging altogether. In relation to scan parameters, our 424 study had better temporal resolution $(33$ vs. $39 \mathrm{~ms})$ and a 425 smaller voxel size $(2.4 \mathrm{~mm} \times 2.4 \mathrm{~mm} v s .3 .3 \mathrm{~mm} \times 2.3 \mathrm{~mm}) \quad 426$ indicating improved spatial resolution.

The effects of left and RV volumes during physical exercise remains controversial. Some data are generally consistent with an enhanced contractile state during supine exercise, but the role of Frank Starling mechanism remains uncertain. In supine exCMR, there are several factors that affect the hemodynamic response. Firstly, exercise in the supine position results in a lower HR response but a greater 
rise in systolic BP, compared with upright exercise $(30,31)$. This results in a similar double product $(\mathrm{HR} \times$ systolic $\mathrm{BP}$ ), which is an index of myocardial oxygenation (32), and a rational for similar detection rates of ischemia between upright and supine stress echocardiography despite a significant lower peak heart rates with supine exercise $(30,33)$. As such, for a given exercise intensity, lower heart rates are expected in supine exercise, compared with upright exercise. Secondly, due to increased afterload in the supine position, it is postulated that end-systolic volumes are near maximal even at rest. As a result, a recent meta-analysis in exCMR studies by Beaudry et al. demonstrated no significant rise in LVEDV, with many studies demonstrating a non-significant decrease with exercise (34). This metaanalysis also demonstrated an exercise rise in LV stroke volume driven by a fall in end-systolic volume. The authors however did not account for one important factor, which is the effect of respiration on cardiac hemodynamics. Claessen et al. (35) elegantly demonstrated the significant effect respiration cycles have on cardiac hemodynamics; with endexpiration resulting in a significant rise in LVEDV and $\mathrm{LV}$ stroke volume and a fall in RVEDV and RV stroke volume, when compared with end-inspiration at numerous levels of exercise. The results of this meta-analysis should therefore be interpreted with caution, as although insightful, it analyses a heterogenous group of exCMR studies including both free-breathing and breath-held acquisitions, in a varied mix of healthy volunteers, endurance athletes and patients with cardiac disease, and who were exercised to different exercise intensities.

The results of this present study are in line with previous studies of supine exercise, showing a decrease in LV $(24,36)$ and RV $(18,23,37)$ EDVs, particularly during later stages of exercise. Similar to previous exCMR studies, we demonstrated no significant rise in stroke volume with exercise (38). Healthy volunteers have been shown to achieve their peak diastolic filling and contractility earlier (24). As a result, LVEDV in healthy volunteers peaked earlier and decreased subsequently. The increase in HR during exercise also reduced diastolic filling time, therefore leading to smaller LV and RV cavity during diastole. It is worth noting that as this study assessed 2 stages of exercise $(55 \%$ and $\left.75 \% \mathrm{HR}_{\max }\right)$ at moderate and high exercise intensities respectively, it is possible our data did not capture the initial LV dilatation described in the Frank Starling mechanism. Indeed, numerous prior investigators $(23,24,35)$ have demonstrated an initial rise in LVEDV and LV stroke volume at early lower exercise stages followed by a fall at later higher intensity stages. However, due to the nature of exCMR studies being performed in small numbers, these changes often do not reach statistical significance. As such it is likely our study did not capture this early rise in LVEDV and LV stroke volume given the exercise stages were performed at moderate and high intensities. This is the first study to confirm clinical feasibility of this exCMR protocol in patients with severe MRegur. Ventricular volumes in patients were unchanged during light and moderate intensity exercise, which is likely a reflection of their relatively deconditioned state and poorer response in terms of myocardial contractility.

CMR MAPSE has been proposed as a simple and easy measure of longitudinal function in healthy volunteers and patients with hypertrophic cardiomyopathy (39). Longitudinal contraction assessed in the form of mitral annular plane systolic excursion (MAPSE) demonstrated that despite similar LV ejection fraction of $>55 \%$, healthy volunteers appeared to have a higher baseline MAPSE than those patients with severe mitral regurgitation; reflecting a better longitudinal contractility of the left ventricle. During exercise at $55 \% \mathrm{HR}_{\max }$, healthy volunteers had an improved longitudinal contractility before the value plateaued at $75 \%$ $\mathrm{HR}_{\max }$. This initial change of improved contractility was not seen in patients with severe mitral regurgitation, potentially reflecting a deconditioned myocardium. These results should however be interpreted with caution in light of the relatively small sample population.

Intra-observer reproducibility of $\mathrm{LV}$ parameters was excellent at all three stages. Similarly, inter-observer reproducibility of $\mathrm{LV}$ parameters was also excellent. Although RVESV measurements were the least reproducible during exercise, the RV ejection fraction and cardiac index were however highly reproducible at all 3 stages. The inter-scan reproducibility was less optimal for LV parameters (CV 5-16\%) and RVESV (CV 11-24\%). The wide interscan variability can possibly be explained by the long 16 weeks scan interval between the 1 st and 2 nd exCMR scans. Although healthy volunteers had no specific exercise training during that period, other factors such as different loading conditions, diet and temperatures could influence cardiac physiology on a day-to-day basis.

This study has highlighted the potential of using 'navigated' image acquisition techniques for the assessment of cardiovascular response during continuous exercise. ExCMR has the potential of providing quantitative cardiac 
indices, whilst offering a direct link between physical activity, symptoms and stress imaging findings. Additionally, it can offer important information such as functional capacity and BP response. The use of exCMR can create new avenues for research and clinical practice, such as stress evaluation of ventricular dysfunction. This is particularly relevant to pathologies of the $\mathrm{LV}$ and $\mathrm{RV}$, and pulmonary circulation that are challenging to assess by other imaging modalities. Further assessment of this ex-CMR protocol is now warranted for assessment of cardiac pathologies where current exercise imaging modalities have been shown to have limitations.

\section{Limitations}

As per all supine exCMR studies, there are general limitations with this approach. Cycling whilst lying in a flat, supine position is an unorthodox form of exercise, and skeletal muscle fatigue may lead to premature test termination (20). Knee-to-bore clearance whilst cycling is also limited by patient height and magnet bore diameter. This study had a maximum participant's height of $188 \mathrm{~cm}$. Furthermore, vigorous respiratory movement can also result in blurring or ghosting of images collated across cardiac cycles. When respiration is performed in the anterior-posterior direction, thus not captured by the navigator in the head-feet direction, the navigator could potentially fail to work. The use of respiratory navigator also causes interruption to steady-state imaging and these signal variations can potentially lead to artifacts, particularly in the systole phase during exercise. Optimal patient preparation, as detailed in the methodology, is therefore vital. Other limitations of exCMR include its inability to be performed in patients with certain implanted devices. Since most CMR acquisitions are acquired over multiple cardiac cycles, arrhythmias such as atrial fibrillation or premature ventricular contractions may pose additional challenges for standard CMR sequences. The study population was small, and the reproducibility should therefore be interpreted with caution. Although highly reproducible, the findings of this study were also not validated against an invasive reference standard. Further work could look into assessing the accuracy of this imaging method against invasive exercise standards (direct Fick method) in deriving cardiac output. This technique was not intended to achieve the $85 \%$ of 'age-predicted maximal heart rate' required for myocardial ischaemia testing purposes, as in-scanner
12 lead ECG monitoring is not feasible, and therefore 576 accurate assessment of ST segment changes during exercise, which may prompt test termination, cannot be performed. The primary aim of this navigated exCMR technique was to assess the serial change in ventricular volumes with exercise as this can serve as an important tool in enabling understanding of physiology in patients with exertional symptoms and structural/congenital heart disease.

\section{Conclusions}

This exercise CMR protocol using a novel application of the free-breathing, multi-shot, navigated imaging method allows simultaneous assessment of the left and RV volumes during continuous exercise. This study demonstrates feasibility of exCMR in patients with mitral regurgitation for the first time. Intra and inter-observer readings were highly reproducible. Clinical feasibility of this protocol suggests a future role in the assessment of patients with exercise-related symptoms.

\section{Acknowledgments}

The authors are grateful for the support and assistance of the radiographers (Margaret Saysell, Gavin Bainbridge, Stephen Mhiribidi, Georgina Casey and Lisa Lewis) and research nurse (Fiona Richards) during this project. Only the abstract of this manuscript ( $<400$ words) has been previously published in Heart 2018;104:A46-7.

Funding: The research was part-supported by the National Institute for Health Research (NIHR) infrastructure at Leeds which has no influence over the contents of this manuscript.

\section{Footnote}

Conflicts of Interest: All authors have completed the ICMJE uniform disclosure form (available at http://dx.doi. org/10.21037/qims-20-117). DMH reports other from Philips, outside the submitted work. GJF reports other from Novartis, during the conduct of the study. The other authors have no conflicts of interest to declare.

Ethical Statement: The study was approved by a local ethics committee (Yorkshire \& The Humber-Leeds West 12/ $\mathrm{YH} / 0551)$ and complied with the Declaration of Helsinki (as revised in 2013). All participants provided written 
informed consent.

Open Access Statement: This is an Open Access article distributed in accordance with the Creative Commons Attribution-NonCommercial-NoDerivs 4.0 International License (CC BY-NC-ND 4.0), which permits the noncommercial replication and distribution of the article with the strict proviso that no changes or edits are made and the original work is properly cited (including links to both the formal publication through the relevant DOI and the license). See: https://creativecommons.org/licenses/by-nc-nd/4.0/.

\section{References}

1. Plana JC, Mikati IA, Dokainish H, Lakkis N, Abukhalil J, Davis R, Hetzell BC, Zoghbi W. A Randomized Cross-Over Study for Evaluation of the Effect of Image Optimization With Contrast on the Diagnostic Accuracy of Dobutamine Echocardiography in Coronary Artery Disease. The OPTIMIZE Trial. JACC Cardiovasc Imaging 2008;1:145-52.

2. Marwick TH, Nemec JJ, Pashkow FJ, Stewart WJ, Salcedo EE. Accuracy and limitations of exercise echocardiography in a routine clinical setting. J Am Coll Cardiol 1992;19:74-81.

3. Thompson RC, Cullom SJ. Issues regarding radiation dosage of cardiac nuclear and radiography procedures. J Nucl Cardiol 2006;13:19-23.

4. Grothues F, Smith GC, Moon JC., Bellenger NG, Collins P, Klein HU, Pennell DJ. Comparison of interstudy reproducibility of cardiovascular magnetic resonance with two-dimensional echocardiography in normal subjects and in patients with heart failure or left ventricular hypertrophy. Am J Cardiol 2002;90:29-34.

5. Ripley DP, Musa TA, Dobson LE, Plein S, Greenwood JP. Cardiovascular magnetic resonance imaging: what the general cardiologist should know. Heart 2016;102:1589-603.

6. Rerkpattanapipat P, Gandhi SK, Darty SN, Williams RT, Davis AD, Mazur W, Clark HP, Little WC, Link KM, Hamilton CA, Hundley WG. Feasibility to detect severe coronary artery stenoses with upright treadmill exercise magnetic resonance imaging. Am J Cardiol 2003;92:603-6.

7. Jekic M, Foster EL, Ballinger MR, Ramanand SV, Simonetti OP. Cardiac function and myocardial perfusion immediately following maximal treadmill exercise inside the MRI room. J Cardiovasc Magn Reson 2008;10:3.

8. Raman SV, Dickerson JA, Jekic M, Foster EL, Pennell
ML, McCarthy B, Simonetti OP. Real-time cine and 671 myocardial perfusion with treadmill exercise stress 672 cardiovascular magnetic resonance in patients referred for 673 stress SPECT. J Cardiovasc Magn Reson 2010;12:41. 674

9. Raman S V., Richards DR, Jekic M, Dickerson JA, 675 Kander NH, Foster EL, Simonetti OP. Treadmill Stress 676 Cardiac Magnetic Resonance Imaging. First In Vivo 677 Demonstration of Exercise-Induced Apical Ballooning. J 678 Am Coll Cardiol 2008;52:1884.

10. Foster EL, Arnold JW, Jekic M, Bender JA, Balasubramanian V, Thavendiranathan P, Dickerson JA, Raman SV, Simonetti OP. An MR-Compatible Treadmill for Exercise Stress Cardiac Magnetic Resonance Imaging. Magn Reson Med 2012;67:880-9.

11. Thavendiranathan P, Dickerson JA, Scandling D, Balasubramanian V, Pennell ML, Hinton A, Raman SV, Simonetti OP. Comparison of treadmill exercise stress cardiac MRI to stress echocardiography in healthy volunteers for adequacy of left ventricular endocardial wall visualization: A pilot study. J Magn Reson Imaging 2014;39:1146-52.

12. Raman SV, Dickerson JA, Mazur W, Wong TC, Schelbert EB, Min JK, Scandling D, Bartone C, Craft JT, Thavendiranathan P, Mazzaferri EL Jr, Arnold JW, Gilkeson R, Simonetti OP. Diagnostic Performance of Treadmill Exercise Cardiac Magnetic Resonance: The Prospective, Multicenter Exercise CMR's Accuracy for Cardiovascular Stress Testing (EXACT) Trial. J Am Heart Assoc 2016;5:e003811.

13. Dymond DS, Foster C, Grenier RP, Carpenter J, Schmidt DH. Peak exercise and immediate postexercise imaging for the detection of left ventricular functional abnormalities in coronary artery disease. Am J Cardiol 1984;53:1532-7.

14. Iliceto S, D’Ambrosio G, Sorino M, Papa A, Amico A, Ricci A, Rizzon P. Comparison of postexercise and transesophageal atrial pacing two-dimensional echocardiography for detection of coronary artery disease. Am J Cardiol 1986;57:547-53.

15. Ranadive SM, Fahs CA, Yan H, Rossow LM, Agliovlastis S, Fernhall B. Heart rate recovery following maximal arm and leg-ergometry. Clin Auton Res 2011;21:117-20.

16. Gusso S, Salvador C, Hofman P, Cutfield W, Baldi JC, Taberner A, Nielsen P. Design and testing of an MRIcompatible cycle ergometer for non-invasive cardiac assessments during exercise. Biomed Eng Online 2012;11:13.

17. Roest AAW, Kunz P, Lamb HJ, Helbing WA, Van Der 
Wall EE, Roos A De. Biventricular response to supine physical exercise in young adults assessed with ultrafast magnetic resonance imaging. Am J Cardiol 2001;87:601-5.

18. Steding-Ehrenborg K, Jablonowski R, Arvidsson PM, Carlsson M, Saltin B, Arheden H. Moderate intensity supine exercise causes decreased cardiac volumes and increased outer volume variations: A cardiovascular magnetic resonance study. J Cardiovasc Magn Reson 2013;15:96.

19. La Gerche A, Claessen G, Van De Bruaene A, Pattyn N, Van Cleemput J, Gewillig M, Bogaert J, Dymarkowski S, Claus P, Heidbuchel H. Cardiac MRI: A new gold standard for ventricular volume quantification during high-intensity exercise. Circ Cardiovasc Imaging 2013;6:329-38.

20. Fletcher GF, Ades PA, Kligfield P, Arena R, Balady GJ, Bittner VA, Coke LA, Fleg JL, Forman DE, Gerber TC, Gulati M, Madan K, Rhodes J, Thompson PD, Williams MA, American Heart Associaton Exercise, Cardiac Rehabilitation and Prevention Committee of the Council on Clinical Cardiology, Council on Nutrition, Physical Activity and Metabolism, Council on Cardiovascular and Stroke Nurseing, and Council on Epidemiology and Prevention. Exercise standards for testing and training: A scientific statement from the American heart association. Circulation 2013;128:873-934.

21. ACSM. ACSM's Guidelines for Exercise Testing and Prescription (10th edition). Wolters Kluwer, 2017:472.

22. Burns J, Sivananthan MU, Ball SG, Mackintosh AF, Mary DASG, Greenwood JP. Relationship between central sympathetic drive and magnetic resonance imagingdetermined left ventricular mass in essential hypertension. Circulation 2007;115:1999-2005.

23. Lurz P, Muthurangu V, Schievano S, Nordmeyer J, Bonhoeffer P, Taylor AM, Hansen MS. Feasibility and Reproducibility of Biventricular Volumetric Assessment of Cardiac Function During Exercise Using Real-Time Radial k-t SENSE Magnetic Resonance Imaging. J Magn Reson Imaging 2009;29:1062-70.

24. Le TT, Bryant JA, Ting AE, Ho PY, Su B, Teo RCC, Gan JSJ, Chung YC, O'regan DP, Cook SA, Chin CWL. Assessing exercise cardiac reserve using real-time cardiovascular magnetic resonance. J Cardiovasc Magn Reson 2017;19:7.

25. Usman M, Atkinson D, Odille F, Kolbitsch C, Vaillant G, Schaeffter T, Batchelor PG, Prieto C. Motion corrected compressed sensing for free-breathing dynamic cardiac MRI. Magn Reson Med 2013;70:504-16.

26. Batchelor PG, Atkinson D, Irarrazaval P, Hill DLG,
Hajnal J, Larkman D. Matrix description of general 766 motion correction applied to multishot images. Magn Reson Med 2005;54:1273-80.

27. Wang Y, Rossman PJ, Grimm RC, Riederer SJ, Ehman RL. Navigator-echo-based real-time respiratory gating and triggering for reduction of respiration effects in three-dimensional coronary MR angiography. Radiology 1996;198:55-60.

28. Danias PG, McConnell MV, Khasgiwala VC, Chuang ML, Edelman RR, Manning WJ. Prospective navigator correction of image position for coronary MR angiography. Radiology 1997;203:733-6.

29. Fletcher GF, Balady GJ, Amsterdam EA, Chaitman B, Eckel R, Fleg J, Froelicher VF, Leon AS, Pina AL, Rodney R, Simons-Morton DA, Williams MA, Bazzarre T. Exercise Standards for Testing and Training: A Statement for Healthcare Professionals From the American Heart Association. Circulation 2001;104:1694-740.

30. Badruddin SM, Ahmad A, Mickelson J, Abukhalil J, Winters WL, Nagueh SF, Zoghbi WA. Supine bicycle versus post-treadmill exercise echocardiography in the detection of myocardial ischemia: A randomized singleblind crossover trial. J Am Coll Cardiol 1999;33:1485-90.

31. Quinn TJ, Smith SW, Vroman NB, Kertzer R, Olney WB. Physiologic responses of cardiac patients to supine, recumbent, and upright cycle ergometry. Arch Phys Med Rehabil 1995;76:257-61.

32. Nelson RR, Gobel FL, Jorgensen CR, Wang K, Wang Y, Taylor HL. Hemodynamic predictors of myocardial oxygen consumption during static and dynamic exercise. Circulation 1974; 50:1179-89.

33. Modesto KM, Rainbird A, Klarich KW, Mahoney DW, Chandrasekaran K, Pellikka PA. Comparison of supine bicycle exercise and treadmill exercise Doppler echocardiography in evaluation of patients with coronary artery disease. Am J Cardiol 2003;91:1245-8.

34. Beaudry RI, Samuel TJ, Wang J, Tucker WJ, Haykowsky MJ, Nelson MD. Exercise cardiac magnetic resonance imaging: A feasibility study and meta-analysis. Am J Physiol Regul Integr Comp Physiol 2018;315:R638-45.

35. Claessen G, Claus P, Delcroix M, Bogaert J, La Gerche A, Heidbuchel $\mathrm{H}$. Interaction between respiration and right versus left ventricular volumes at rest and during exercise: A real-time cardiac magnetic resonance study. Am J Physiol Heart Circ Physiol 2014;306:H816-24.

36. Furukawa K, Nishida K, Yamada C, Niki S, Sugihara H, Kohno Y, Katsume H, Ijichi H, Kitamura H, Kunishige H. Left ventricular size and performance during graded supine 
exercise in normal subjects. Jpn Heart J 1983;24:503-14.

37. Mols P, Huynh CH, Naeije N, Ham HR. Volumetric response of right ventricle during progressive supine exercise in men. Am J Physiol 1991;261:H751-4.

38. Steeden JA, Atkinson D, Taylor AM, Muthurangu V. Assessing vascular response to exercise using a combination of real-time spiral phase contrast MR and noninvasive blood pressure measurements. J Magn Reson Imaging $\quad 820$ 2010;31:997-1003.

39. Taylor RJ, Moody WE, Umar F, Edwards NC, Taylor TJ, 822 Stegemann B, Townend JN, Hor KN, Steeds RP, Mazur 823 W, Leyva F. Myocardial strain measurement with feature- 824 tracking cardiovascular magnetic resonance: Normal values. Eur Heart J Cardiovasc Imaging 2015;16:871-81.

Cite this article as: Chew PG, Swoboda PP, Ferguson C, Garg P, Cook AL, Ibeggazene S, Brown LAE, Craven TP, Foley JR, Fent GJ, Saunderson CE, Higgins DM, Plein S, Birch KM, Greenwood JP. Feasibility and reproducibility of a cardiovascular magnetic resonance free-breathing, multi-shot, navigated image acquisition technique for ventricular volume quantification during continuous exercise. Quant Imaging Med Surg 2020;10(9):1837-1851. doi: 10.21037/qims-20-117 


\section{Chamber exCMR cine images}

Baseline

HR 84

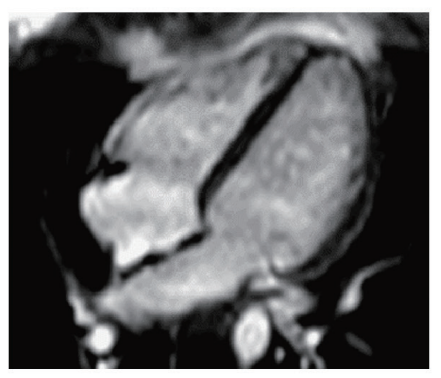

Short axis stacks of

exCMR cine images
$55 \% \mathrm{HR}_{\max }$

HR 106

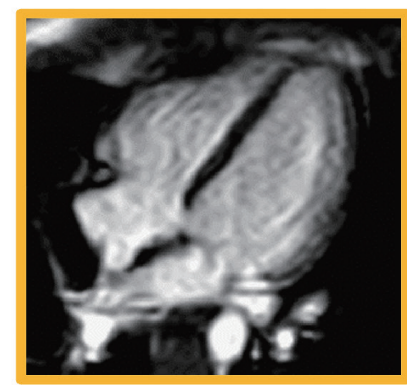

$75 \% \mathrm{HR}_{\text {max }}$

HR 138

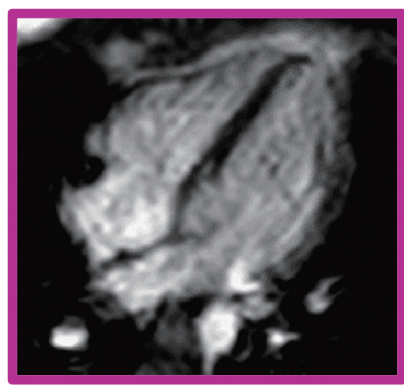

Baseline HR 84

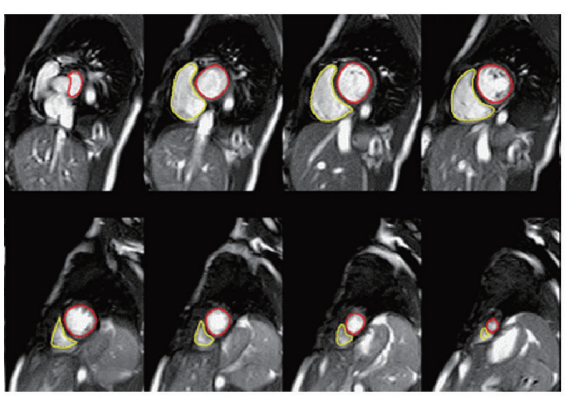

$75 \% \mathrm{HR}_{\text {max }}$

HR 138
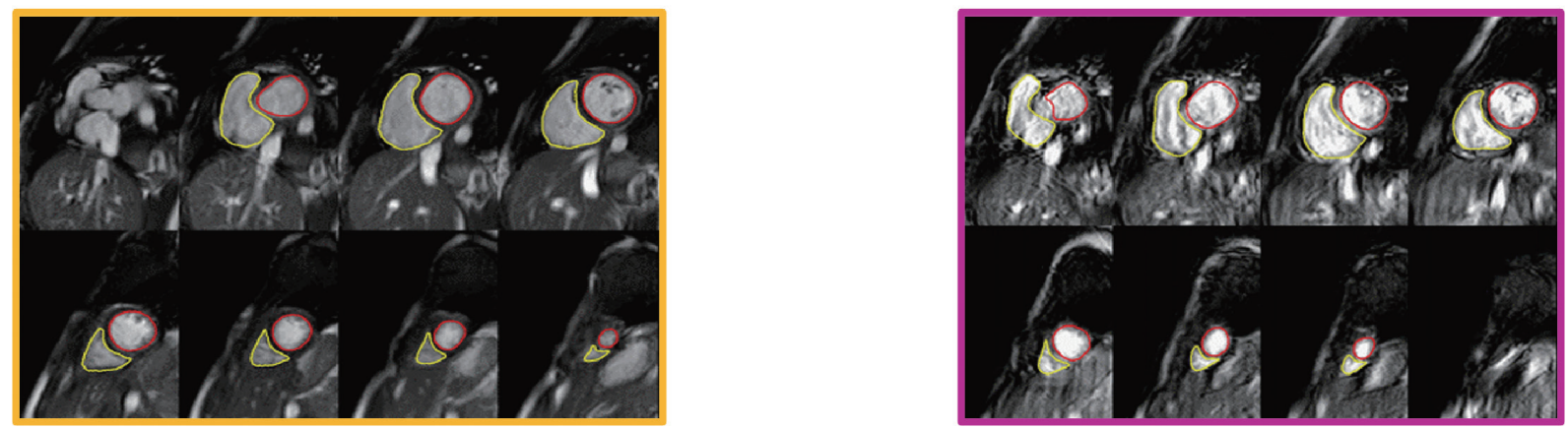

Figure S1 Illustration of navigated exCMR cine images from one volunteer. Cine images at baseline, $55 \% \mathrm{HR}_{\max }$ and $75 \% \mathrm{HR}_{\max }$. Heart rate at time of imaging was 84, 106 and 138 bpm, respectively. 